\title{
Opinion piece: Myths surrounding the investigation and treatment of erectile dysfunction
}

\author{
1. Partner participation is necessary during the investigation \\ and treatment of erectile dysfunction
}

Richard Casey MD FRCSC, Editor-in-Chief

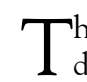
he realm of sexual dysfunction (SD), in particular erectile dysfunction (ED), has changed significantly over the past 20 years. ED was originally considered to be a nonorganic entity and, accordingly, much of the treatment by physicians was focussed on the psychosocial aspects of the patient and his partner. With increased focus on research in this area, there is now a better understanding of the pathophysiology of ED and effective treatments have been developed, including oral medications. As a result, there has been a paradigm shift from nonorganic roots to specific organic etiologies such as diabetes and atherosclerotic vessel disease.

A majority of health care providers completed their education before the release of the first phosphodiesterase 5 inhibitor, sildenafil. Has the change in our understanding of ED now led to attitude changes in our approach to the problem? Do we have a firm understanding of the impact of the role of oral agents, especially with the advent of newer and potentially more effective therapies?

The knowledge base surrounding SD will continue to increase over time as will the number of new therapy options we can offer our patients. Can we take the experience with ED into the area of female SD? Should we change the way we address sexual problems in our practice overall? Can urologists continue to provide effective therapy to their patients despite such a 'penocentric' approach? We will explore a few of the controversial conceptions surrounding the triage and treatment of ED in the postphosphodiesterase 5 era.

Does the partner need to be involved in the ED treatment plan?

We have all heard the story of the spouse who, after her husband receives a sildenafil prescription, complains to her physician, "I liked him better when he was impotent ... he tried harder. Now he gets his erection and wants to use it right away."

While this scenario is entirely believable, does it suggest that we should always ask for the partner to be present for at least one of the office visits? In my clinical experience, most partners prefer to stay at home and not become intimately involved in the assessment and treatment plans. This is despite the fact that the partner can provide significant knowledge to the treating physician on the functional status of the penis in question and will be affected by the success of the treatment. My experience is not unique and, in fact, similar observations have been published in the past few years.

In one example, Lee et al (1) examined the attitudes of patients and their partners toward joint sessions and psychological counselling as a means of treating ED. After an initial consultation (conducted at the Human Sexuality Clinic, Kingston, Ontario, 100 consecutive patients were asked to return with their partners. Sixty per cent did not return with their partners. Of the partners who did participate, the general attitude was that the patients' ED difficulties were the patients' problem, not the partners'. The partner's attitude was often one of indifference or a preference for no further sexual intercourse.

In a separate study by Lewis et al (2) involving oral medication treatment, patient and partner satisfaction with sildenafil were measured using the ED inventory of treatment satisfaction questionnaire. A total of 247 patients with ED were treated in a randomized, double-blind parallel-group, multicentre study conducted through outpatient clinics. The partner completion rate was only $21 \%$ and $25 \%$ for sildenafil and placebo, respectively. The reason for this low rate of completion, particularly as part of a clinical study, is not known. However, it suggests that the involvement of the partner in ED patient treatment may not be as active as we would expect.

It is acknowledged that most outpatient settings are not appropriate for conjoint consultations. Certainly in Canada, where remuneration issues tend to streamline consultation efforts, the trend is toward treatment of the ED in isolation from the partner. Family physicians are better suited to include relationship issues in their treatment decisions, particularly if the ED patient's partner is also a member of his/her practice. But is the treatment of the patient without the partner likely to sabotage the outcome?

The 'invested partner', defined as a sexually normal individual within a couple invested in a relationship with a dysfunctional subject, traditionally was part of the treatment plan (3). It stands to reason that the use of vacuum devices, intracorporal injection therapy, MUSE or penile prosthesis implantation is best introduced with the invested partner's knowledge because concealment is difficult and partner support is recommended for these treatments. Oral therapy has changed this

The Male Health Centres, Oakville, Ontario

Correspondence: Dr Richard W Casey, 407-1235 Trafalgar Road North, Oakville Ontario L6H 3P1. Telephone 905-338-3130, fax 905-338-3150, e-mail drcasey@on.aibn.com 
because the erectile response is returned to its expected path and partner participation occurs through sexual stimulation. Then, is it less important that the partner be involved in this particular phase of the disease management?

It should be noted that female sexual functioning may be altered as a result of her partner experiencing ED. Renshaw et al (4) showed that the female partner of a man with ED was more likely to experience sexual disorders such as orgasmic dysfunction, vaginismus, dyspareunia and impaired sexual interest; female SD preceded the onset of ED in less than $10 \%$ of the cases (4). Treatment for the ED alone may not lead to the resumption of successful sexual intercourse. In these cases, counselling and treatment of the female partner may be necessary to repair the damage done by the ED.

Is there a subset of ED patients who can be managed in isolation? Conversely, should we insist on couple management with certain individuals?

Riley et al (5) reviewed case records from 128 consecutive men presenting with ED whose partners attended on the first or second visit. The duration of ED varied from several months to 40 years. Interestingly, over one-half of the couples had not experienced any mutual sexual activity for about 2.5 years. Over $80 \%$ of the men and only $20 \%$ of the female partners considered intercourse important. ED frequently results in complete avoidance of sexual activity; because men are often the initiators of sexual activity, it was suggested that there is no sexual activity among couples where the man has ED. How often have we heard the following phrase when treating men: "Why should I start something I can't finish?" Longstanding ED often results in significant relationship damage that will not be repaired by a hard penis. These patients require either short or long term supportive counselling and we should require the partner's attendance at some point during the consultative process. In the Canadian model, family physicians should be encouraged to refer both members of the ED couple (to a urologist/sex therapist?) for assessment in these situations.
There are instances where the onset of ED will not have the same impact on a couple's relationship. Postprostatectomy ED, for example, is often accepted as part of the disease 'package' and can be successfully resolved, in most instances, without the partner's participation in the treatment plan. It is difficult to find instances where couples therapy would not be beneficial, but certainly postsurgical ED is often handled with a more physiological approach, largely because the partner understands the etiology and does not feel, in part, responsible for the lack of tumescence. Similar attitudes are present in the partners of diabetic patients.

ED sufferers will continue to attend their physicians without their partners and it is paramount that we recognize those couples at risk. It would be appropriate to investigate to some degree the patient-partner relationship and insist on partner attendance if the initial treatment plan is unsuccessful. Partner involvement during the diagnostic and educational aspects of the treatment program is often sufficient to resolve many of the outstanding issues. Unresolved issues can then be referred to a more formal setting. In my opinion, it is not necessary to insist that our ED patients bring along their partners during the initial stages of consultation. However, if a reasonable treatment plan is unsuccessful, then assessment of the partner is mandatory.

\section{REFERENCES}

1. Lee JC, Surridge DHC, Morales A, Heaton JPW. Erectile dysfunction: The perspectives of patients and partners on counseling. J Sex Reprod Med 2002:2:11-5.

2. Lewis R, Bennett CJ, Borkon WD, et al. Patient and partner satisfaction with Viagra (sildenafil citrate) treatment as determined by the erectile dysfunction inventory of treatment satisfaction questionnaire. Urology 2001;57:960-5.

3. Derogatis LR, Meyer JK, Gallant BW. Distinctions between male and female invested partners in sexual disorders. Am J Psych 1977;134:385-90.

4. Renshaw DC. Coping with an impotent husband. Ill Med J 1981;159:29-33.

5. Riley A, Riley E. Behavioral and clinical findings in couples where the man presents with erectile disorder. A retrospective study. IJCP 2000;54:220-4. 